\title{
Section III
}

\section{Evaluating Practices to Improve Teaching}

No one gets out of graduate school without learning that the proper emphasis in "critical thinking" is on the word critical: if you don't think otherwise, you must not be thinking. Not surprising, then, is it that we are regularly, inevitably asked "What is the evaluation?" "Does it work?" "What is the effect?" "Yes, but compared to what?" "What is the level of significance? Surely at least the .05 level!"

Maybe. But probably not all the time, we mumble. Yet no collection of resources would be complete without some attention to assessment of our activities. The three articles which comprise this section address some common concerns: teaching effectiveness, course evaluation and the impact of developmental interventions.

Between one fourth and half of undergraduate education is conducted by graduate teaching assistants. Bob Menges and Jeremy Wilson note that this is not only fact, but a common source of complaint. What Bob and Jeremy show us, however, is that at least at Northwestern T.A.'s do a pretty good job in the view of undergrads.

Another old chestnut in the assessment game is course evaluation. At Syracuse Bob Diamond and Ricard Sudweeks have outlined a general recipe for approaching evaluation of college courses, wherein the focus is on course objectives, design and impact - usually the institutional and administrative issues that we must struggle with as developers and teachers, but also concerns relevant to students, even if at some remove. 
The last article by Mary Deane Sorcinelli is an application of classical experimental design to measure the personal development she sees as one outcome of the widespread teaching consultation process originated almost a decade ago at the University of Massachusetts. Though her sample is small and the significance will be thus questioned, the issue is critical to all of us. Is there any worthwhile change in teaching, learning or administrating that does not have an essential personal (affective, emotional, identity) component? "All learning is affect," Joe Katz has claimed. Even if we quibble, we must be more intentional and conscious about our effectiveness with the affective. 